\title{
Current Status of Heterojunction Bipolar and High-Electron Mobility Transistor Technologies
}

\author{
Dimitris Pavlidis
}

Solid State Electronics Laboratory, Department of Electrical Engineering and Computer Science, The University of Michigan, Ann Arbor, MI 48109-2122, USA

\begin{abstract}
The current status of Heterojunction Bipolar and High-Electron Mobility Transistor technology is reviewed. Applications include analog and digital circuits with focus on high power and optical communications for HBT's and millimeter-wave low-noise and power modules for HEMT's. Material choices, designs, technology, reliability issues and circuit demonstrations are addressed.
\end{abstract}

\section{HETEROJUNCTION BIPOLAR TRANSISTOR TECHNOLOGY}

Heterojunction Bipolar Transistor (HBT) Technology has advanced during the recent years and experimental characteristics have been demonstrated in terms of power, noise and high-frequency performance. The technology has matured to the degree where some laboratories have also been successful in producing HBT monolithic circuits for various applications, very often by letting their fabrication lines accessible to external designers in a "foundry-like" mode. The most advanced material system used in HBT's is GaAs/AlGaAs. Other very promising heterostructures include GaInP/GaAs and $\mathrm{InP} / \mathrm{InGaAs}$ or InAlAs/InGaAs, the latter two being lattice-matched to InP rather than GaAs substrates. This section reviews the various designs, technologies and characteristics of HBT's and discusses recent advances in these areas.

\subsection{MATERIAL CHOICES AND DESIGNS}

As already mentioned, the traditional and most advanced approach for HBT technology is AlGaAs/GaAs. This material is much more advanced than any other choice regarding growth and fabrication procedures and is more compatible, with processes in GaAs-FET lines. Another interesting option is GaInP/GaAs. GaInP has negligible DX-center problems compared to AlGaAs, and allows almost "trap-free" operation. The GaInP/GaAs heterointerface merits, however, special attention since compound

Work supported in part by NASA (Contr. No. NAGW-1334), URI-ARO (Contr. No. DAAL 03-87-K-0007) and Texas Instruments (Contr. No. 109106476). 
formation of GaInAsP or rough interface characteristics may limit the benefits drawn by "bulk" GaInP. Another crucial feature is the large valence-band discontinuity $\left(\Delta \mathrm{E}_{v}\right)$ of $\mathrm{GaInP} / \mathrm{GaAs}(0.22-0.28 \mathrm{eV})$ and $\Delta \mathrm{E}_{c}$ discontinuity of $\sim 0.20 \mathrm{eV}$ which compared to Al$\mathrm{GaAs} / \mathrm{GaAs}$ result in higher injection efficiency and energy carrier launching, respectively.

Another option is the InP/InGaAs material system which offers a number of advantages compared with $\mathrm{AlGaAs} / \mathrm{GaAs}$ such as: 1) higher $\Delta \mathrm{E}_{v}(0.37 \mathrm{eV}$ vs. $0.13 \mathrm{eV})$ and therefore larger injection efficiency. 2) Larger $\Gamma$-L valley separation in InP and InGaAs $(\sim 0.5 \mathrm{eV})$ compared to $\mathrm{GaAs}(0.28 \mathrm{eV})$ which lead to sustained velocity overshoot characteristics in the collector 3$)$ smaller surface recombination velocity $\left(1 \times 10^{3} \mathrm{~cm} / \mathrm{s} \mathrm{in} \mathrm{InGaAs}\right.$ vs. $10^{6} \mathrm{~cm} / \mathrm{s}$ in GaAs and 4) smaller bandgap ( $\left.\mathrm{E}_{g}\right)$ for the InGaAs base and thus smaller turn-on voltage and power consumption in digital circuits. This system is not, however, without disadvantages. In GaAs collectors result for example in lower breakdown voltage than GaAs due to their small bandgap. The use of InP collectors (double HBT designs) alleviates this problem due to the larger $\mathrm{E}_{g}$, but unfortunately introduces a conduction spike at the B-C junction which degrades electron transport and speed.

InAlAs/InGaAs offers another alternative with very similar features as $\mathrm{InP} / \mathrm{InGaAs}$. Furthermore, it's large $\Delta \mathrm{E}_{c}(\sim 0.53 \mathrm{eV})$ and the associated spike can be used as launching ramp of electons for quasi-ballistic transport in the base. Recent work by $\mathrm{Hu}$ et al. [1] showed, however, that the spike may result in fast carrier transfer to the $\mathrm{X}$ or $\mathrm{L}$ valleys which are characterized by lower effective velocity. E-B grading by $\operatorname{In}_{0.53}\left(\mathrm{Ga}_{1-x} \mathrm{Al}_{x}\right) \mathrm{As}$ with $\mathrm{x}$ varying from 1.0 to 0.6 turns out to be optimum for fast transport and cutoff frequencies of $270 \mathrm{GHz}$ are predicted using such designs.

The most popular growth technique used for HBT's is Metallorganic-Vapor Pressure Epitaxy (MOVPE). Other approaches such as MBE and MOMBE have, however, also been used. N-type doping is in most cases achieved by Si while base P-doping has been attempted by Zn (MOVPE), Be(MBE) and more recently carbon. Carbon appears to be very attractive for achieving extremely high doping levels (values up to $10^{20} \mathrm{~cm}^{-3}$ have been reported) while at the same time providing the possibility of reducing diffusion problems from $\mathrm{E}$ to $\mathrm{B}$, thus enhancing reliability and permitting good performance control within the design specifications.

The collector design has drawn considerable attention. Proper tailoring of the electric field in the B-C space-charge-region (SCR) constrains the eletrons from being transferred to upper valleys and allows velocity overshoot extending over a longer distance. This can be achieved by replacing the conventional $\mathrm{n}^{-}$collector design by other layers such as $\mathrm{p}^{-}$ (inverted electric field), $\mathrm{i}-\delta \mathrm{p}^{+}$or simply i for InP-HBT's (undoped collector), $\delta \mathrm{n}^{+}-\mathrm{n}^{-}-\delta \mathrm{p}^{+}$ (collector launcher [2]) or $\mathrm{p}^{-} \mathrm{n}^{-}$(for good breakdown-speed compromise in InP/InGaAs HBT's [3]).

On the emitter side, tunneling designs have been proposed [4] where carriers are injected at very high energy and thus speed to the base, while at the same time a large valence-band discontinuity can be preserved at the E-B junction; a thin ( $50 \AA)$ barrier can be used for this purpose. GaInP is particularly attractive for such designs [5] since it 
provides the possiblity of large $\Delta \mathrm{E}_{v}$ without the need for extremely large $\mathrm{Al}$ compositions as in the case of AlGaAs.

\subsection{TECHNOLOGY AND FACTORS DICTATING PERFORMANCE}

Almost all fabrication approaches converge nowadays in a self-aligned approach for the base with respect to the emitter terminals. Reactive-Ion-Etching (RIE) is employed for reaching the base using $\mathrm{BCl}_{3}$ and $\mathrm{Ar}$ (for AlGaAs) or $\mathrm{CH}_{4} / \mathrm{H}_{2} / \mathrm{Ar}$ (for InP) gases and followed by slight wet etching to remove the plasma induced damage and create the necessary overhang for good lift-off. One of the major issues is device isolation and reduction of the B-C parasitic capacitance. This is particularly crucial for InGaAs collector HBT's where ion-implantation techniques are not applicable. Wet etching is the only obvious solution in this case and makes the need for planarization techniques by polymides absolutely essential. Various implant species such as oxygen, protons and helium can be used for isolating GaAs-based devices. In addition to offering planarity, ion-implantation also allows a reduced area of parasitic $\mathrm{B}-\mathrm{C}$ region and thus smaller parasitic capacitance and charging time.

Etching selectivity of the emitter over the base layer is particularly useful for controling the process of reaching the base. Although not the case for most AlGaAs/GaAs designs (unless high Al composition emitters or a mismatched InGaAs base is used), both GaInP/GaAs and InP/InGaAs offer such features.

Table 1: Summary of Experimentally Demonstrated Maximum Frequency Limits of HBT's

\begin{tabular}{cccccc} 
Design & Material & $\begin{array}{c}\text { Emitter Size } \\
\left(\mu \mathrm{m}^{2}\right)\end{array}$ & $\begin{array}{c}\mathrm{f}_{T} \\
(\mathrm{GHz})\end{array}$ & $\begin{array}{c}\mathrm{f}_{\max } \\
(\mathrm{GHz})\end{array}$ & Laboratory/Year \\
\hline $\begin{array}{c}\text { SHBT } \\
(\text { Ballistic C) }\end{array}$ & AlGaAs/GaAs & $1.6 \times 9.6$ & 171 & 70 & NTT-1990 \\
\hline SHBT & AlGaAs/GaAs & $1.25 \times 9.0$ & 75 & 175 & Rockwell 1988 \\
\hline SHBT & InP/InGaAs & $3.6 \times 3.6$ & 165 & 100 & AT\&T-1989 \\
\hline SHBT & AlInAs/InGaAs & $3.5 \times 3.5$ & 91 & 40 & AT\&T-1989 \\
\hline $\begin{array}{c}\text { SHBT } \\
(\text { Graded E) }\end{array}$ & AlInAs/InGaAs & $2.0 \times 5.0$ & 138 & 78 & Hughes 1992
\end{tabular}

The reliability of HBT's seems to be primarily impacted by dopant diffusion from the base to the emitter. The observed degradation occurs when the device is biased while it is being temperature stressed and is absent for unbiased HBT's. The likely mechanism responsible for these effects is a field-assisted diffusion of positively charged interstitial $\mathrm{Be}$ atoms from the GaAs base to the AlGaAs emitter. This causes the formation of a conduction-band spike which is evidenced by a higher $V_{B E}$ turn-on voltage. Larger space-charge recombination and reverse-hole injection is also present as evidenced by a higher base current. Various approaches such as increased As/Ga flux ratio and reduced substrate temperature [6] have been proposed to reduce interstitial generation. Another very promising possibility is the use of Carbon dopants. Ohmic contact degradation of alloyed AuGe contacts is also affecting reliability. 
The identification of the sources reponsible for the low-frequency noise characteristics of HBT's has drawn significant attention since most reported devices do not appear to have the expected low transition frequencies in the $1 / \mathrm{f}$ spectra. Recent studies on the impact of the low-frequency noise to the phase noise of HBT oscillators showed that the former affects the frequency response of the oscillator noise but under certain conditions the upconversion characteristics play a more predominant role than the $1 / \mathrm{f}$ noise [ 7$]$.

\subsection{ELECTRICAL CHARACTERISTICS}

AlGaAs HBT's have demonstrated very high frequency of operation with cutoff frequencies exceeding $170 \mathrm{GHz}$ for $\mathrm{f}_{T}$ and $220 \mathrm{GHz}$ for $\mathrm{f}_{\max }$. They are therefore well suited for high-frequency analog and also high-speed digital applications. Equally high $\mathrm{f}_{T}$ values have also been achieved by InP/InGaAs HBT's. Table 1 summarizes the HBT maximum frequency characteristics demonstrated experimentally for various material systems. Very high power-added efficiencies exceeding $68 \%$ with $12 \mathrm{~dB}$ gain have also been demonstrated at X-band [8] and power levels up to $5.3 \mathrm{~W}$ at $\mathrm{X}$-band have been reported [9]. A number of analog and digital monolithic circuits have also been built and showed very promising electrical performance. Insertion to optical communication systems is being considered both in the U.S. and Japan for broadband digital networks.

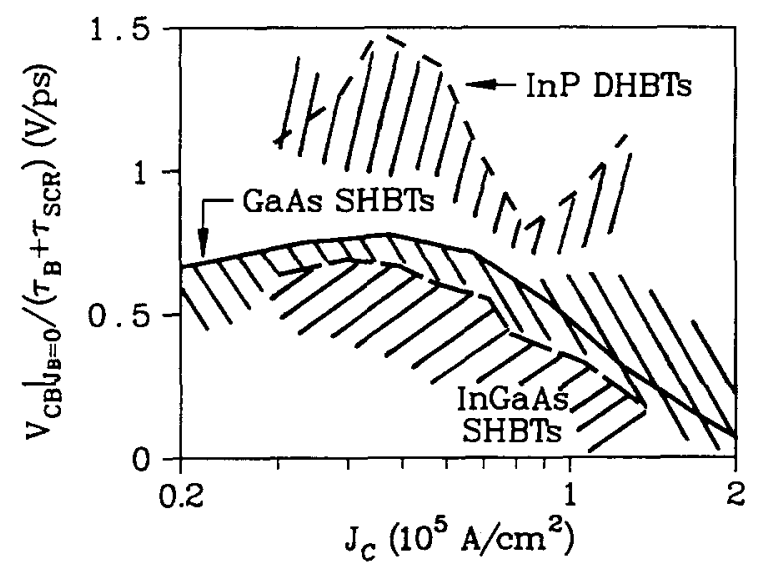

Figure 1: Speed-Breakdown Tradeoffs of AlGaAs/GaAs and InP/InGaAs HBT's as evaluated at the University of Michigan [3]

Although InP/InGaAs HBT's are expected to perform much better in terms of highfrequency characteristics, they are unfortunately handicapped from the point of view of maximum operating voltage. due to the low breakdown voltage of the InGaAs collector. For certain applications it is, however, interesting to consider the speed-breakdown tradeoffs of the device. Figure 1 shows such an evaluation for GaAs and InP-based HBT's using $\left.\mathrm{V}_{C B}\right|_{J_{B=0}} /\left(\tau_{B}+\tau_{S C R}\right)$ as a figure of merit [9]. Here $\left.\mathrm{V}_{C B}\right|_{J_{B=0}}$ is the voltage associated with the base current reversal effect indicative of breakdown and $\tau_{B}+\tau_{S C R}$ the total transit time. A Drift-Diffusion and Monte-Carlo simulator were used for calculating the two parameters respectively. The results show that as far as speed-breakdown is con- 
cerned there is no particular advantage to be gained out of single InP-HBT's. However, the figure of merit is highest for double InP-HBT suggesting superior performance with such designs.

\section{HIGH-ELECTRON-MOBILITY TRANSISTOR TECHNOLOGY}

High-Electron-Mobility Transistors (HEMT's) can be built with a variety of material systems and have demonstrated the ultimate performance in terms of high-frequency operation capability among other three terminal devices. Noise and power characteristics are also excellent compared to other devices and offer unique advantages especially at millimeter-wave frequencies. The traditional approach is based on $\mathrm{AlGaAs} / \mathrm{GaAs}$ but more recently other materials such as GaInP/GaAs and InAlAs/InGaAs have emerged. The former provides the possibility of excellent cryogenic operation [10], while the latter is the best choice for low-noise and millimeter-wave applications.

Current trends in HEMT technology focus on applications in low-noise front ends and advanced Microwave Monolithic and Optoelectronic Integrated Circuits (MMICs' and OEIC's). Strained (pseudomorphic) designs continue drawing the attention for improved carrier confinement and channel transport properties. Other materials such as Sb-based compounds are finally under exploration.

\subsection{MATERIAL CHOICES AND DESIGNS}

GaAs-based HEMT's are traditionally built using AlGaAs donor layers of $\sim 22 \%$, Alcontent in order to reduce the presence of DX-centers. To overcome the problem of low $\Delta \mathrm{E}_{c}$ in such designs a pseudomorphic InGaAs channel with $\sim 20 \%$ In can be used. Devices built on such materials have a higher maximum frequency of operation $\left(f_{T}\right.$ of $\sim 150$ $\mathrm{GHz}$ instead of $\sim 100 \mathrm{GHz}$ for lattice-matched designs) and superior noise characteristics. This approach is also compatible with the well established and mature GaAs technology.

GaInP offers an interesting alternative to the AlGaAs donor layers. It's use is compatible with GaAs-technology and allows absence of threshold voltage shift and I-V collapse at cryogenic temperatures due to minimum content of DX-centers [10]. Pseudomorphic designs can also be realized with this system. Further work is, however, required to improve the material and heterointerface quality which is currently limiting the microwave performance.

Lattice matched and pseudomorphic designs can finally be realized on InP substrates using the InAlAs/InGaAs system. Here, not only is the use of AlGaAs totally eliminated, but the resulting high $\Delta E_{c}$ provides good carrier confinement. This, combined with good transport properties in the high In-content channel, results in very low noise figures (NF

$=0.8 \mathrm{~dB}, \mathrm{G}_{A}=8.7 \mathrm{~dB} @ 63 \mathrm{GHz}[11]$ and high frequency capability $\left(\mathrm{f}_{\max }=450 \mathrm{GHz}\right.$ [12]).

Growth techniques used in HEMT technology include MBE, MOVPE and CBE. In the case of InP-based HEMT's the latter two techniques offer unique advantages due to their 
better compatibility with optoelectronic applications requiring phosphorous containing compounds. The latter can also be used for obtaining channel and buffer layers with high breakdown voltage characteristics as necessary for power applications. Although MOVPE and CBE approaches are in general considered not as well suited for abrupt heterointerfaces as $\mathrm{MBE}$, recent results at the University of Michigan showed excellent and almost comparable performance for InP-based HEMT's [13] [14].

\subsection{TECHNOLOGY AND ELECTRICAL PERFORMANCE}

Shorter gate-lengths offer the possibility of enhanced microwave performance and have therefore motivated the development of reliable E-beam technologies. Currently achieved gate-lengths are in the range of $0.1 \mu \mathrm{m}$ using bi- and tri-layer processes and mushroom gate profiles for reduced gate resistance. $\mathrm{f}_{T}$ improvement up to $300 \mathrm{GHz}[15]$ has been observed with $650 \AA$ gate-lengths, inspite of the presence of short channel effects.

Self-aligned technologies for the ohmic contacts with respect to the gate permit minimum access parasitics and improved performance. Gate offsetting results in reduced gateto-drain capacitance and enhanced $\mathrm{f}_{\max }$ performance combined with higher breakdown capability. Recent efforts allowed the combination of the self-aligned and offset $\Gamma$-gate features [14] and demonstrated with the help of CBE-grown InAlAs/InGaAs HEMT's the possibility of $\mathrm{f}_{\max }$ ratios which are as high as 2.7 . f $\max$ values reaching $310 \mathrm{GHz}$ have been achieved using this approach, as shown by Figure 2.

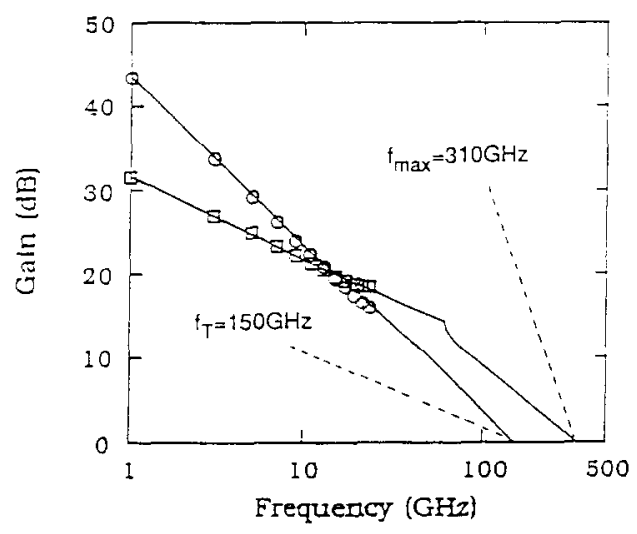

Figure 2: High $\mathrm{f}_{\text {max }}$ InAlAs/InGaAs CBE-HEMT using the self-aligned $0.15 \mu \mathrm{m}$ offset $\Gamma$-gate technology developed at the University of Michigan

Gate recess still consists a major limitation for controlled and reproducible characteristics. The conventional wet etchants used in most cases are non-selective and those that show selectivity have in most cases very fast etch rates. This results in very large lateral etch and performance degradation. The recommended solution is dry etching and work is in progress in this area with a view to process optimization for controlled etching with minimum surface damage.

A number of undesirable features have also been reported for InAlAs/InGaAs HEMT's. These include the kink-effect observed in the I-V characteristics, a high output conduc- 
tance, low breakdown voltage, excess-gate leakage and sidegating effects. Various approaches have been proposed to suppress these problems and these include the use of (i) superlattice or low-temperature buffers for reduced kink- effect, (ii) double-heterojunction designs, p-doped buffers and undoped cap to minimize the output conductance, (iii) multi-heterojunction designs, undoped cap, double recess and P-containing channels to improve the breakdown, (iv) higher Al content Schottky layers and Pt Schottky metals to reduce leakage and ( $\mathrm{v}$ ) planar technologies by ion-implantation isolation to reduce sidegating.

Noise performance improves steadily up to millimeter-wave frequencies as evidenced experimentally, by using pseudomorphic instead of lattice-matched GaAs-based HEMT's. InP-based devices offer even lower noise figures. The power characteristics of HEMT's are also excellent, as can be stated by the data of Figure 3 [16]. Multi-heterojunction designs and doped-channel pseudomorphic HEMT approaches offer the best way to enhance the power and power-added efficiency. The results of Figure 3 show that PMHEMT's offer the best choice and clear benefits can be gained from them, especially at millimeter-wave frequencies.

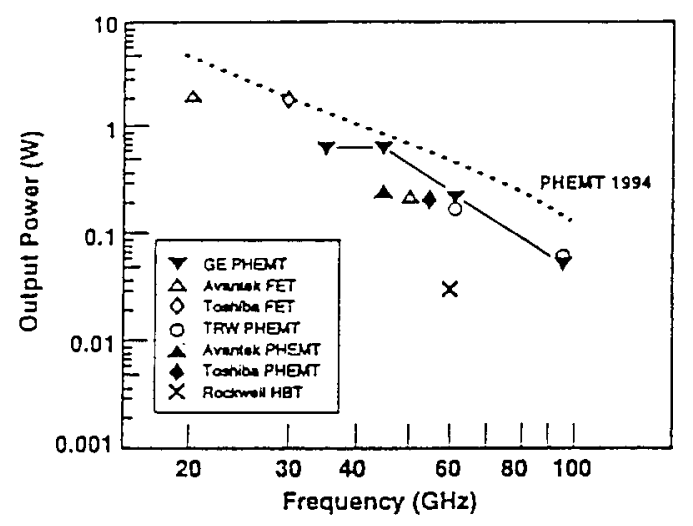

Figure 3: Power characteristics of HEMT's and HBT's (Courtesy of G.E. [14])

Although some doubts may be expressed regarding the reliability of pseudomorphic approaches, recent results [17] showed that the DC lifetime exceeds $10^{6}$ hours at $150^{\circ} \mathrm{C}$ baseplate temperature and that the pseudomorphic channel does not contribute to any additional degradation mechanism. The devices used for these tests were AlGaAs/InGaAs HEMT's passivated by $\mathrm{Si}_{s} \mathrm{~N}_{4}$. Although equally good reliability performances are claimed for InAlAs/InGaAs devices, degradation effects have been reported for this material system [18] and work is in progress in various laboratories to understand the related problems. Various attempts are also being made in the area of surface passivation of such devices.

\subsection{MONOLITHIC CIRCUIT APPLICATIONS}

MMIC's have been built using both GaAs and InP-based HEMT technology and excellent performance has been demonstrated up to millimeter-wave frequencies. These achievements using InP-based devices include monolithic amplifiers up to at least W- 
band, oscillator-doubler modules up to D-band [19], doublers with signal output at $180 \mathrm{GHz}$ and mixers with conversion gain at $94 \mathrm{GHz}$ [20]. Ultra-wide band amplifiers operating from 5 to $100 \mathrm{GHz}$ [21] have also been demonstrated using this material system.

\section{ACKNOWLEDGEMENT}

The author is grateful to the students, faculty and staff of his department as well as to the industry laboratories which shared their recent program results in the area of advanced heterojunction devices.

\section{REFERENCES}

[1] J. Hu, D. Pavlidis and K. Tomizawa, IEEE Trans. on El. Dev., Vol. 39, No. 6, June 1992 [2] T. Ishibashi, H. Nakajima, H. Ito, S. Yamahata and Y. Matsuoka, 48th Annual Device Research Conference, Paper VIIB, 3, 1990

[3] H-F. Chau, D. Pavlidis, J. Hu and K. Tomizawa, Proc. of 4th InP \& Rel. Mat. Conf., Newport, R.I., U.S., pp. 410-413, April 1992

[4] J. Xu and M. Shur, IEEE Elec. Dev. Lttrs., Vol. 7, p. 416, 1986

[5] T. Lauterbach, W. Pletschen and K.H. Bachem, IEEE Trans. on El. Dev., Vol. 39, No. 4, April 1992

[6] D.C. Streit et al., IEEE El. Dev. Lttrs, Vol. 12, No. 9, pp. 471-473, Sept. 1991

[7] M. Tutt, D. Pavlidis, A. Khatibzadeh and B. Bayraktaroglu, IEEE MTT-S Int. Microwave Symp. Digest, Albuquerque, U.S., pp. 727-730, June 1992

[8] M.F. Chang et al., Int. J. of High Speed Electr., 1, 245, 1991

[9] B. Bayraktaroglu, M.A. Khatibzadeh and R.D. Hudgers, Dig. IEEE Micr. \& MM-Wave Mon. Circ. Symp., pp. 43-46, 1990

[10] Y-J. Chan, D. Pavlidis, M. Razeghi and Omnes, IEEE Trans. on El. Dev., Vol. 37, No. 10, pp. 2141-47, October 1990

[11] U.K. Mishra, A.S. Brown, L. Jelloian, L.H. Hackett and M.J. Delaney, IEEE El. Dev. Lttrs., Vol. 9, No. 1, pp. 41-43, 1988

[12] P.M. Smith et al. Proceedings of 2nd InP \& Rel. Mat. Conf., pp. 39-43, 1990

[13] G.I. Ng, D. Pavlidis, Y. Kwon, T. Brock, J.I. Davies, G. Clarke and P.K. Rees, Proc. of 4th Annual InP \& Rel. Mat. Conf., Newport, R.I., U.S., pp. 18-21, April 1992

[14] Y. Kwon, T. Brock, G.I. Ng, D. Pavlidis, G.O. Munns, M.E. Sherwin and G.I. Haddad, Proc. of 4th InP \& Rel. Mat. Conf., Newport, R.I., U.S., pp. 18-21, April 1992

[15] L.D. Nguyen, A.S. Brown, M.A. Thompson, L.M. Jelloian, L.E. Larson and M. Matloubian, IEEE El. Dev. Lttrs., Vol. 13, No. 3, pp. 143-145, 1992

[16] P.M. Smith, P.C. Chao and J.M. Ballingall, , IEEE Princeton Sarnoff Symposium, Princeton, N.J., U.S., March 1992

[17] W.-W. Hu, P.-C. Chao, M.Y. Kao, P. Ho, R.J. Finke and A.W. Swanson, , 1991 GaAs IC Symp. Digest, pp. 191-194, Oct. 1991

[18] Dumas and D. Lecrosnier, Proc. of 4th Int. Conf. on InP and Rel. Mat., Newport, R.I., U.S., pp. 214-217, April 1992

[19] Y. Kwon, D. Pavlidis, P. Marsh, G.I. Ng and T. Brock, G.O. Munns and G.I. Haddad, To be presented at 1992 IEEE GaAs Symp., Miami, Florida, October 1992

[20] Y. Kwon, D. Pavlidis, P. Marsh and G.I. Ng, To appear in IEEE Trans. on MTT

[21] R. Majidi-Ahy et al., IEEE Trans. on MTT, Vol. 38, No. 12, pp. 1896-1993, 1990 